\title{
Lim1 Is Essential for the Correct Laminar Positioning of Retinal Horizontal Cells
}

\author{
Ross A. Poché, ${ }^{1}$ Kin Ming Kwan, ${ }^{2}$ Mary A. Raven, ${ }^{4}$ Yasuhide Furuta, ${ }^{3}$ Benjamin E. Reese,${ }^{4,5}$ and Richard R. Behringer ${ }^{1,2}$ \\ ${ }^{1}$ Program in Developmental Biology, Baylor College of Medicine, and Departments of ${ }^{2}$ Molecular Genetics and ${ }^{3}$ Biochemistry and Molecular Biology, \\ University of Texas, M. D. Anderson Cancer Center, Houston, Texas 77030, and ${ }^{4}$ Neuroscience Research Institute and ${ }^{5}$ Department of Psychology, \\ University of California at Santa Barbara, Santa Barbara, California 93106
}

\begin{abstract}
Although much is known about the transcriptional regulation that coordinates retinal cell fate determination, very little is known about the developmental processes that establish the characteristic laminar architecture of the retina, in particular, the specification of neuronal positioning. The LIM class homeodomain transcription factor Lim1 (Lhx1) is expressed in postmitotic, differentiating, and mature retinal horizontal cells. We show that conditional ablation of Lim1 results in the ectopic localization of horizontal cells to inner aspects of the inner nuclear layer, among the retinal amacrine cells. The ectopic cells maintain a molecular phenotype consistent with horizontal cell identity; however, these neurons adopt a unique morphology more reminiscent of amacrine cells, including a dendritic arbor positioned within the inner plexiform layer. All other retinal cell populations appear unaltered. Our data suggest a model whereby Lim1 lies downstream of horizontal cell fate determination factors and functions cell autonomously to instruct differentiating horizontal cells to the appropriate laminar position in the developing retina. This study is the first to describe a cell type-specific genetic program that is essential for targeting a discrete retinal neuron population to the proper lamina.
\end{abstract}

Key words: differentiation; horizontal cell; lamina; migration; plasticity; retina

\section{Introduction}

With only seven classes of cells organized into three discrete layers, the retina lies in stark contrast to the vast array of cell types in the mature brain and has thus proven to be a powerful model system to address fundamental questions regarding CNS development. Over the last two decades, the study of retinal development has been particularly successful in elucidating mechanisms of neuronal fate determination. Specifically, much progress has been made in revealing which intrinsic and extrinsic factors instruct multipotent retinal progenitor cells to follow a specific fate determination program (Cepko, 1999; Livesey and Cepko, 2001; Hatakeyama and Kageyama, 2004). However, very little is known about what factors regulate laminar positioning once a particular cell type has been specified. To date, most vertebrate retinal lamination mutants exhibit a global disorganization of the retinal architecture. These phenotypes are often the result of disruptions in cell adhesion molecules, such as integrins and cadherins, or cell polarity factors (Malicki et al., 1996, 2003; Masai et al., 2003;

\footnotetext{
Received Sept. 4, 2007; revised 0ct. 22, 2007; accepted Nov. 1, 2007.

This work was supported by National Eye Institute Grants T32 EY07102 (R.A.P.), R01 EY11087 (B.E.R.), and R01 EY013128 (Y.F.), and the Ben F. Love Endowment (R.R.B.). We thank Artur Kania and Thomas Jessell for the Lim7 ${ }^{\text {lacz/+ }}$ mice; Sheldon Rowan and Connie Cepko for the Chx10-GFPCre ${ }^{\text {tg/++}}$ mice; Xiugian Mu, Steven Wang, Mengqing Xiang, Yuko Akiyama, William Klein, and Beniot de Crombrugghe for antibodies; James Morgan for the Pep19 antibody; Jane Johnson for the Ptf1a antibody; and Gabriella D’Arcangelo and Randy Johnson for critical reading of this manuscript. We also thank Hank Adams and Aya Wada for advice on microscopy.

Correspondence should be addressed to Dr. Richard R. Behringer at the above address. E-mail: rrb@mdanderson.org.

K. M. Kwan's present address: Department of Biology, The Chinese University of Hong Kong, Hong Kong SAR. DOI:10.1523/JNEUROSCI.4046-07.2007

Copyright $\odot 2007$ Society for Neuroscience $\quad 0270-6474 / 07 / 2714099-09 \$ 15.00 / 0$
}

Koike et al., 2005; Fu et al., 2006; Seymour et al., 2007), affecting multiple cell types. These data do not address whether the various classes of retinal neurons employ similar or different mechanisms to achieve proper lamination. Furthermore, considering the overwhelming evidence that combinatorial transcription factor codes direct cell fate specification, it is unclear whether similar transcriptional networks orchestrate the subsequent lamination. The overall picture of retinal development, from fate specification to lamination and synaptogenesis, likely involves a complex transcriptional regulatory cascade.

Horizontal cells (HCs) are a class of retinal interneuron that modulates signal transmission between the photoreceptors and bipolar cells. Within the last few years, significant progress has been made in understanding the early fate-determining factors for this cell type. The homeodomain transcription factor Prox1, was reported to be an intrinsic factor required for commitment to the HC fate (Dyer et al., 2003). Subsequently, the forkhead/ winged helix transcription factor Foxn 4 was also shown to be essential for HC genesis, because Foxn4-null retinas exhibit a complete loss of HCs (Li et al., 2004). Very recently, the basic helix-loop-helix transcription factor Ptfla was found to lie genetically downstream of Foxn4. Mice with null mutations in Ptfla, exhibit a phenotype similar to the Foxn4-null mice (Fujitani et al., 2006). Despite the recent progress in elucidating the factors regulating HC fate specification, virtually nothing is known about factors regulating later aspects of $\mathrm{HC}$ development. Postmitotic, differentiating HCs are distributed throughout the neuroepithelium and thus require a localization mechanism(s) to direct appropriate terminal laminar positioning (Hinds and Hinds, 1979; Liu et al., 2000; Edqvist and Hallbook, 2004). Once localized to 
the correct layer, HCs differentiate in response to their afferent innervation and establish functional connections with synaptic partners (Raven et al., 2007). Evidence of LIM class homeodomain transcription factor Lim1 (Lhx1) expression in differentiating HCs (Liu et al., 2000) suggests that Lim1 plays a role in these later developmental processes downstream of fate determination. Using a conditional knock-out approach, we show that, in a cellautonomous manner, loss of Lim1 function results in the ectopic positioning of HCs in an otherwise normally laminated retina. Furthermore, we show that the mutant cells undergo a morphological transformation reminiscent to that of an amacrine cell, yet retain the molecular identity of a HC. This study is the first to describe a mouse model that uncovers a cell typespecific developmental genetic program that is essential for proper targeting of an entire retinal neuron population to the appropriate layer.

\section{Materials and Methods}

Mouse strains. Lim1 $1^{\text {lacZ/+ }}$ (Kania et al., 2000), Lim1 $1^{\text {flox/flox }}$ (Kwan and Behringer, 2002), and Six3-Cre ${ }^{\text {tg/+ }}$ (Furuta et al., 2000) mice were maintained on a C57BL/6J, 129/SvEv mixed genetic background. Chx10-GFPCre $e^{t g /+}$ (Rowan and Cepko, 2004) mice were maintained on a FVB, 129/SvEv, C57BL/6J, and SJL mixed genetic background.

PCR genotyping. Liml lacz/+, Lim1 $1^{\text {flox/flox }}$ Six3-Cre $e^{t g /+}$, and Chx10-GFPCre $e^{\text {tg/+ }}$ mice were genotyped as described previously (Furuta et al., 2000; Kwan and Behringer, 2002; Kobayashi et al., 2004; Rowan and Cepko, 2004).

Histology. X-gal staining and immunofluorescence on frozen retinal sections was performed as described previously (Ohtoshi et al., 2001; Wang et al., 2001). Immunofluorescence on thick sections cut on a Vibratome was also performed for confocal microscopy, as described previously (Raven et al., 2007).

Generation of a polyclonal anti-Lim1 antibody. Through a contract with Bethyl Laboratories, we generated a Lim1-specific antibody. A synthetic peptide with an amino acid sequence of CLGGLDHPLPGHHPSSEA was purified and then injected into a goat as immunogen. The resulting antibodies within the hyperimmune antisera were affinity purified.

Retinal markers. A table of all antibodies including dilutions and references can be found within supplemental Table 2 (available at www.jneurosci.org as supplemental material).

\section{Results}

The LIM class homeobox gene Lim1 is required for head, female reproductive tract, and kidney development (Shawlot and Behringer, 1995; Kobayashi et al., 2004, 2005). Lim1 is also widely expressed throughout the developing and adult CNS, but the precise roles of $\operatorname{Lim} 1$ within neuronal populations remain poorly defined. Interestingly, Lim1 transcripts and protein were localized to differentiating and mature retinal HCs (Liu et al., 2000; Edqvist and Hallbook, 2004). However, because of the headless Lim1-/- phenotype (Shawlot and Behringer, 1995), analysis of the function of Lim1 in the retina has not previously been addressed.
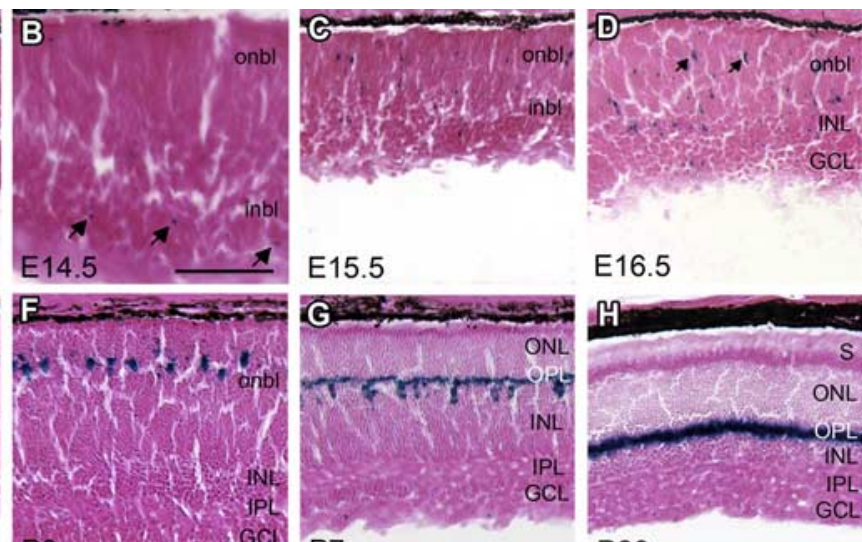

E16.5
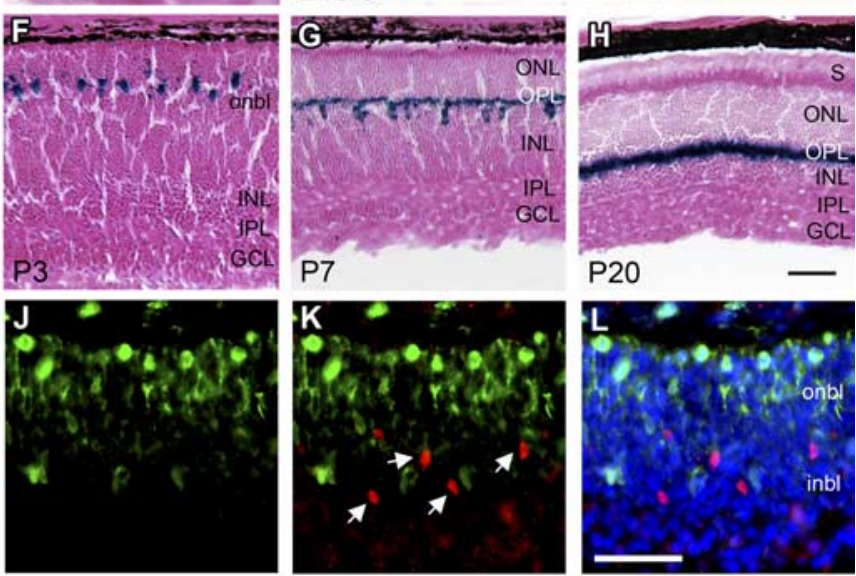

P20
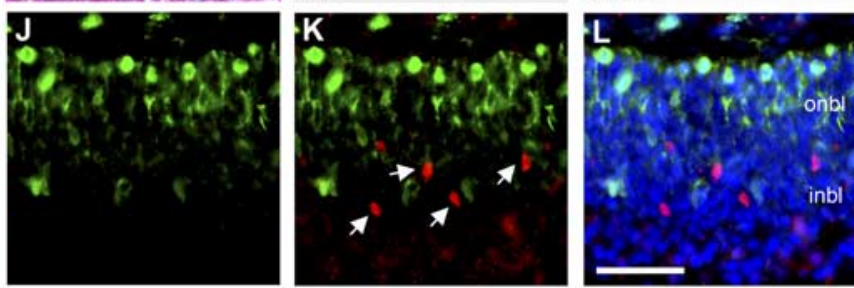

Lim1 expression

\section{HC genesis}

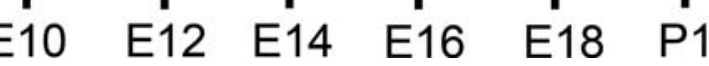

Figure 1. Expression pattern of the Lim 1-lacZ allele during mouse retinogenesis. $\boldsymbol{A}-\boldsymbol{H}$, Retinal sections from the indicated developmental stages were stained for $\beta$-galactosidase activity. All images represent the central retina at the level of the optic nerve. Lim 1-lacZ expression is first detected at E14.5 in discrete puncta within the inbl (indicated by arrows in $\boldsymbol{A}$ and $\boldsymbol{B}$ ). I- $\boldsymbol{L}, \mathrm{E} 15.5$ genesis versus $\operatorname{Lim} 1$ expression. Scale bars: (in $\boldsymbol{B}, \boldsymbol{H}) \boldsymbol{A}-\boldsymbol{H}, 50 \mu \mathrm{m}$; (in $\boldsymbol{L}) \boldsymbol{I}-\boldsymbol{L}, 50 \mu \mathrm{m}$. In this and all subsequent figures, a minimum of three mice were examined at each age and for each genotype presented.

The temporal and spatial pattern of Lim1 expression suggests a biological role in horizontal cell development subsequent to fate determination

To gain additional insight into the exact temporal and spatial expression of $\operatorname{Lim} 1$ in the developing mouse retina, we analyzed the expression of a Lim1-tau::lacZ null, knock-in allele (Kania et al., 2000). X-gal staining of retinas heterozygous for the Lim1lac $Z$ allele revealed $\beta$-galactosidase (lacZ) activity beginning on embryonic day 14.5 (E14.5) (Fig. $1 A, B$, arrows). Because mouse HCs become postmitotic between E11 and E16 (Young, 1985a), this observation suggests that $\operatorname{Lim} 1$ functions in later aspects of $\mathrm{HC}$ development and is not involved in progenitor proliferation or HC fate determination (Fig. $1 M$ ). Interestingly, at E14.5, discrete lacZ+ puncta are localized to the vitreal side of the developing retina adjacent to the prospective ganglion cell layer (GCL). By E15.5, there is an upregulation of Lim-lacZ expression and lacZ $+\mathrm{HCs}$ exhibit more scattering throughout the retina (Fig. 1C). By E16.5, this pattern becomes more obvious with a small proportion of the lacZ + cells locating to the prospective HC layer within the outer neuroblastic layer (onbl) (Fig. 1D, arrows). By E18.5, in the central retina, most lacZ + HCs have settled into the prospective $\mathrm{HC}$ layer, thus forming the characteristic $\mathrm{HC}$ monolayer (Fig. $1 \mathrm{E}$ ). In the intermediate and peripheral retina, varying degrees of lac $\mathrm{Z}+\mathrm{HC}$ scattering are observed with most scattering confined to the periphery (data not shown). Because the retina develops in a central to peripheral manner, these 


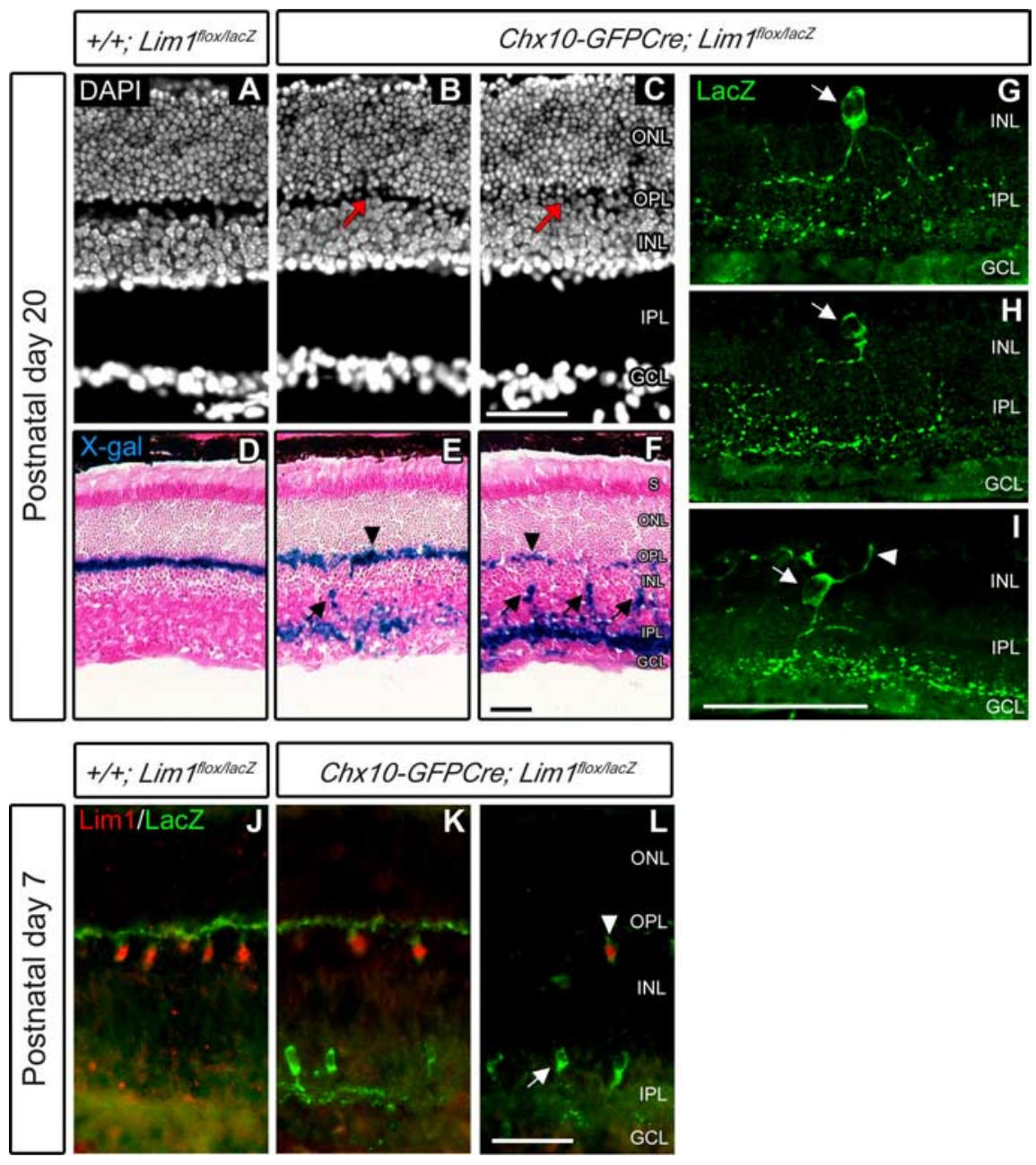

Figure 2. Gross abnormalities of the Lim 1 conditional mutant retina. $A-C, A$ DAPI $\left(4^{\prime}, 6^{\prime}\right.$-diamidino-2-phenylindole) stain of $\mathrm{P} 20$ retinal sections reveals collapse of the conditional mutant $\mathrm{OPL}$ (compare $\boldsymbol{A}$ with $\boldsymbol{B}$ and $\boldsymbol{C}$ ). $\boldsymbol{D}-\boldsymbol{F}, \beta$-Galactosidase activity was assessed at P20. In Lim 1 conditional mutant retinas $(\boldsymbol{E}, \boldsymbol{F})$, ectopic Lim1-lacZ + cells are observed projecting into the IPL and stratifying adjacent to the GCL. In control retinas, Lim1-lacZ is confined to the horizontal cell layer in projections stratifying within the OPL (D). G-I, LacZ immunofluorescence reveals the morphology of the ectopic lacZ + cells. Colabeling for lacZ and Lim1 uncovers mosaic recombination of the $\operatorname{Lim} 1$ locus. $\boldsymbol{J}-\boldsymbol{L}$, All ectopic lacZ + cells $(\boldsymbol{K}, \boldsymbol{L})$ fail to express Lim1, whereas all normally localized cells ( $\boldsymbol{J}-\boldsymbol{L}$, arrowhead) maintain $\operatorname{Lim} 1$ expression. To illustrate the range of mosaic Cre-mediated recombination, two separate conditional mutant retinas are shown $(\boldsymbol{B}, \boldsymbol{C}, \boldsymbol{E}, \boldsymbol{F}, \boldsymbol{K}, \boldsymbol{L})$. Note that the samples shown in $\boldsymbol{C}, \boldsymbol{F}$, and $\boldsymbol{L}$ appear more severely affected than $\boldsymbol{B}, \boldsymbol{E}$, and $\boldsymbol{K}$. Scale bars: (in $\boldsymbol{F}) \boldsymbol{D}-\boldsymbol{F}, 50 \mu \mathrm{m}$; (in $\boldsymbol{C}, \boldsymbol{I}, \boldsymbol{L}) \boldsymbol{A}-\boldsymbol{C}, \boldsymbol{G}-\boldsymbol{I}, \boldsymbol{J}-\boldsymbol{L}, 50 \mu \mathrm{m}$.

data suggest that these less developed regions still contain migratory HCs that have yet to settle in the prospective HC layer. By postnatal day $3(\mathrm{P} 3)$, all regions of the retina contain lacZ $+\mathrm{HCs}$ that are confined to a single monolayer within the onbl (Fig. $1 F$ ). $\mathrm{By} \mathrm{P} 7$, in the central and intermediate retina, lac $\mathrm{Z}+\mathrm{HC}$ processes have stratified to the developing outer plexiform layer (Fig. $1 G$ ), whereas more peripheral cells differentiate a stratified morphology a few days later, to achieve their characteristic laminar pattern observed in maturity (Fig. $1 \mathrm{H}$ ). Additionally, by colabeling with an antibody for the cell cycle marker, Ki67, and an antibody we generated against Lim1, we were able to confirm that Lim1 + HCs fail to express Ki67 and are indeed postmotic (Fig. $1 I-L$ ). These results are in accordance with data from the chicken retina showing that migrating Lim $1+$ cells are indeed postmotitic (Edqvist and Hallbook, 2004). Additionally, by colabeling with antibodies against lacZ and Lim1, we observed that Lim1 protein is expressed in the nuclei of all Lim1-tau::lacZ+ HCs (Fig. 2J). Together, these data show that Lim1 is expressed in postmitotic, migratory as well as mature HCs and that the Lim1-lacZ allele correctly recapitulates the HC-specific expression of the endogenous Lim1 protein (Liu et al., 2000).

\section{The Lim 1 conditional mutant retina displays deficits in horizontal cell positioning}

To investigate the role of Lim1 in retinal development, we generated conditional mutants using two different retina-specific Cre lines, Six3-Cre (Furuta et al., 2000) and Chx10-CreGFP (Rowan and Cepko, 2004). Six3-Cre first shows retina-specific recombinase activity at E9.5 and by E12.5 displays robust activity in retinal progenitor cells throughout the neuroblastic layer (Furuta et al., 2000). Chx10-CreGFP becomes active at E10.5 and exhibits robust Cremediated recombination shortly thereafter leading to fate mapping across the entire mature retina (Rowan and Cepko, 2004). By crossing these lines to mice carrying the ROSA26R Cre reporter allele, we independently verified that both lines exhibit widespread Cre-mediated recombination before the earliest detected expression of Lim1 (data not shown). Importantly, both lines produced Lim1 conditional mutant phenotypes virtually identical with one another. The data presented here are primarily from the Chx10-CreGFP line, which was used to generate mice of the genotype Chx10-Cre Cim $^{+/ t g}$ flox/lacZ . These mice carried the Cre transgene in addition to one copy of the Lim-floxed and one copy of the null Lim1-lacZ knock-in allele. Thus, X-gal staining or lacZ immunohistochemistry could be used to positively identify Lim1null HCs. Chx10-Cre-mediated Lim1 conditional mutants were obtained at the expected Mendelian ratios. Mutants were viable and fertile with no obvious gross ocular or behavioral abnormalities.

Cryosectioning and subsequent nuclear stain of P20 conditional mutant retinas revealed that the outer plexiform layer (OPL) was reduced in thickness, being almost completely collapsed in some regions (Fig. $2 A-C$, arrows). The nuclear layers, as well as the inner plexiform layer (IPL), of the conditional mutant retinas were of similar thickness to the controls, suggesting a relatively specific phenotype associated with Lim1 deletion.

To assess the fate of the Lim1-null cell population, X-gal staining was performed on P20 retinal tissue. Surprisingly, in the mutants, many Lim1-lacZ+ cells are ectopically localized to inner regions of the inner nuclear layer (INL), mostly within the amacrine cell stratum. Furthermore, these mispositioned lacZ+ cells project into the IPL rather than into the OPL, producing multistratifying dendritic arbors most profusely within the inner regions of the IPL adjacent to the ganglion cell layer (GCL) (Fig. $2 D-F$, arrows). To better visualize the morphology of the ectopic lacZ + cells, lacZ immunofluorescence was performed. Ectopically localized lacZ + cells have a teardrop-shaped soma from which an elaborate dendritic tree extends into the IPL (Fig. 2G-I) 
reminiscent of amacrine cells. Occasionally, single apically directed processes are also seen extending from the ectopic soma to the OPL (Fig. 2I, arrowhead).

In all Lim1 conditional mutants analyzed, some lacZ + HCs remain in their proper location within the INL adjacent to the OPL (Fig. 2E,F, arrowheads). This observation was thought to reflect the previously documented mosaic activity of the Cre-recombinase (Rowan and Cepko, 2004; Johnson et al., 2006). By colabeling with antibodies against lacZ and Lim1, we were able to show that all normally localized lacZ + HCs in the conditional mutant mice were indeed Lim1 + , whereas all ectopic lacZ+ cells were Lim1-negative (Fig. $2 J-L)$. Thus, mosaic activity of the Chx10Cre recombinase revealed a cellautonomous role for Lim 1 in Lim1-lacZ+ cell laminar positioning.

\section{Ectopic Lim1-lacZ + cells are misplaced horizontal cells}

Because of the change in localization and aberrant morphology of the Lim1-null lacZ + retinal neurons, a thorough assessment of ectopic cell identity was deemed crucial. In light of our data showing that ectopic cells retain expression of the Lim1lac $Z$ allele (Fig. $2 E, F$ ), an allele specifically expressed in HCs (Fig. 1), these cells are reasonably presumed to be misplaced but are otherwise correctly fated to become HCs. To make a more complete distinction between a cellular positioning defect versus a cell fate change, a battery of retinal markers was used in anti-lacZ coimmunofluorescence experiments. In all samples analyzed, the ectopic lacZ + cells consistently colabeled with the $\mathrm{HC}$ marker Calbindin. Notably, the Calbindin labeling in the ectopic lacZ + cells was of comparable intensity with that of normally placed HCs (Fig. $3 A-F$, arrowheads and inset). These data suggest that the misplaced lacZ+ cells are $\mathrm{HC}$ in identity. Likewise, Doublecortin (Dcx) has been reported to be expressed in migratory HCs, and labeling for Dcx at P2 shows that ectopic lacZ + cells retain Dcx expression (Fig. $3 G, J$ ). These data are also consistent with the ectopic cells being horizontal in identity, but because Calbindin is also normally expressed in amacrine and ganglion cells (albeit at significantly lower levels) (Haverkamp and Wassle, 2000), and because Dcx is also expressed in postmigratory amacrine and ganglion cells (Lee et al., 2003), these markers were considered consistent with, but not conclusive, for establishing $\mathrm{HC}$ identity.

Because of the change in the dendritic morphology and polarity of these ectopic cells, reminiscent of amacrine cells (Fig. 2G$I)$, and in light of the close lineage relationship of amacrine and HC progenitors (Dyer et al., 2003; Li et al., 2004; Fujitani et al., 2006), we analyzed a variety of amacrine cell markers for coexpression in the ectopic lacZ + cells. As expected, all of the ectopic lacZ + cells colabel with Prox1, known to be expressed in HCs, bipolar cells, and AII amacrine cells (Dyer et al., 2003). In contrast, these cells never colabel with the AII amacrine cell-specific marker Dab1 (Rice and Curran, 2000; Haeseleer et al., 2004) (Fig. $3 H, I, K, L)$. If the ectopic lacZ+ cells were AII amacrine cells, we would expect the cells to express both Prox 1 and Dab1. Thus, we conclude that the ectopically localized lacZ + cells are not AII amacrine cells. In every conditional mutant sample analyzed, the additional amacrine cell markers Calretinin, Pep19, Isl-1, P57, ChAT, GABA, and GLYT-1 never colabeled any ectopic lacZ+ cells (Fig. 4; supplemental Fig. 8, available at www.jneurosci.org as supplemental material).

Subsequent analysis of bipolar cell-specific markers Chx10GFPCre, anti-Chx10, and anti-PKC $\alpha$ revealed that the ectopic lacZ + cells are not bipolar cells (supplemental Fig. 8, available at www.jneurosci.org as supplemental material) (data not shown). Furthermore, colabeling was never seen with markers consistent with photoreceptors, ganglion cells, or Müller glial cells (supplemental Fig. 8, available at www.jneurosci.org as supplemental 


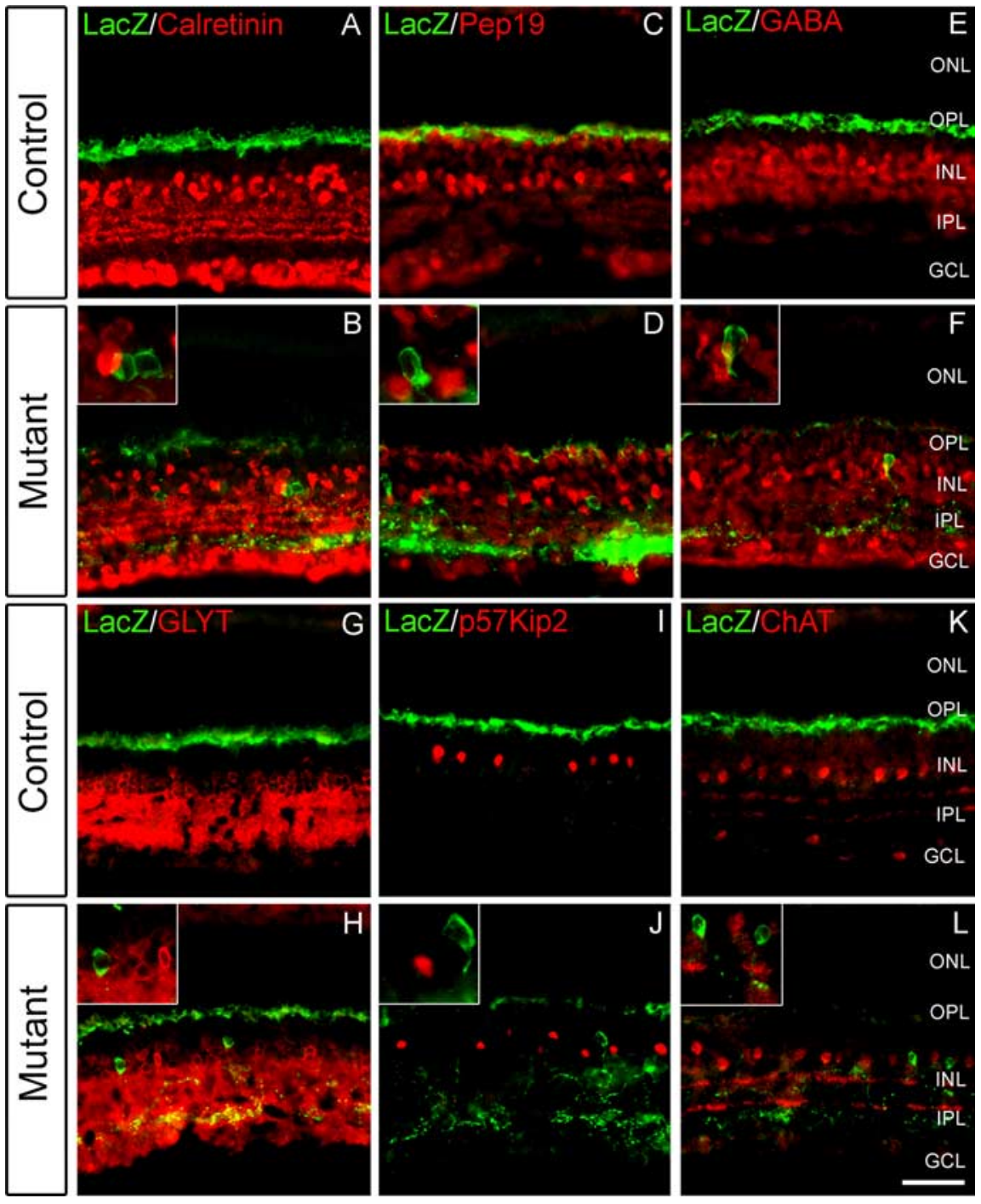

Figure 4. Ectopic LacZ + cells fail to express markers of amacrine cell identity. $A-L$, Ectopic lacZ + cells never express any of the six indicated amacrine cell markers. For the conditional mutant samples, note the complete lack of colocalization within the insets $(\boldsymbol{B}-\boldsymbol{F}, \boldsymbol{H}-\boldsymbol{L})$. Scale bar, $50 \mu \mathrm{m}$.

material). In total, we tested 23 retinal markers for coexpression with Lim1-lacZ (Table 1) and observed colabeling consistent only with HC identity. These data strongly suggest that the ectopic lacZ + cells are HCs that have failed to locate to their proper laminar position.

To more conclusively show that Lim1-null cells are indeed properly fated to become HCs, we also performed Lim1-lacZ/ Prox1 coimmunofluorescence on E16.5 and E18.5 mutant retinas. Prox 1 has been shown to regulate the exit of retinal progenitor cells from the cell cycle and to be necessary and sufficient for HC fate determination (Dyer et al., 2003). It is not yet clear whether Prox 1 is expressed in retinal progenitor cells that give rise to other cells types such as amacrine cells. In this study, we use Proxl not as a horizontal cell precursor-specific marker, but rather as marker consistent with appropriate specification to the horizontal cell fate. At E16.5, control HCs coexpress Lim1-lacZ and Prox 1 and are scattered throughout the retina with a small proportion reaching the onbl where the HC layer will eventually form (Fig. 5A-C). In the Lim 1 mutant retinas, most of the HCs have accumulated near the vitreal side of the retina and do not appear to be translocating apically as in the control (Fig. 5D-F).
Most importantly, in these mutant cells, Lim1-lacZ/Prox coexpression clearly persists (Fig. 5F, arrows). At E18.5, control Lim1-lacZ+ HCs still express Proxl and have now begun to form the $\mathrm{HC}$ monolayer within the onbl (Fig. $5 G-I$ ). In the mutant retinas, a clear failure of monolayer formation is observed, yet these cells continue to express Proxl (Fig. 5J-L, arrows). Another transcription factor, $P t f 1 a$, has been shown to determine $\mathrm{HC}$ and amacrine cell fates and lies genetically upstream of Prox 1 in the $\mathrm{HC}$ fate determination pathway (Fujitani et al., 2006). Through coimmunofluorescence, we sought to determine whether embryonic Lim1-null cells also express Ptf1a. Interestingly, at both E16.5 and E18.5, control Lim1-lacZ+ HCs do not express Ptfla (supplemental Fig. 9A-H, inset; available at www.jneurosci.org as supplemental material). Thus, it is likely that Ptfla is transiently expressed in the earliest stages of HC fate determination and turns off before Lim1 expression is initiated. The remaining observed Ptfla expression is likely within the amacrine cell lineage. We also analyzed E12.5, E14.5, and E16.5 control and Lim1 mutant retinas for Ptf1a expression. At E12.5, both the control and mutant retinas clearly express a few, scattered Ptfla + cells in accordance with the published expression profile (supplemental Fig. 9I,J, available at www.jneurosci.org as supplemental material). Thus, in the mutant retina, Ptfla expression is properly initiated. At E14.5 and E16.5, the mutant retinas also continue to express Ptfla at levels relative to the control (supplemental Fig. $9 K-R$, available at www.jneurosci.org as supplemental material). In total, these data suggest that migratory Lim1-null, Lim1-lacZ+ cells are properly fated to the $\mathrm{HC}$ identity, but fail to locate to the prospective HC layer, thereby resulting in the $\mathrm{HC}$ ectopia observed in the adult mutant retinas.

Mispositioned horizontal cells are evident shortly after the initiation of Lim 1 expression

A time course analysis of the $\operatorname{Lim} 1$ conditional mutant phenotype revealed that misplaced lacZ+ HCs first appear at E15.5 (Fig. $6 A, B)$. This time point corresponds to a day after Lim1-lacZ is first detectable. Moreover, at E15.5, the ectopic lacZ+ cells appear to be more intensely stained with Lim1-tau::lacZ, thereby suggesting these cell are larger than the lacZ + cells in the control. It is possible that the cell morphology is already affected at this early stage and this attribute may allude to either a cause or an effect of the cells having failed to reach the prospective HC layer. By E16.5, more ectopic lacZ + cells accumulate within the inner neuroblastic layer (inbl) (Fig. 6C,D), and by E18.5, a clear failure of lacZ $+\mathrm{HCs}$ to reach the prospective HC layer is evident (Fig. $6 E, F)$. Through Lim1/Lim1-lacZ coimmunofluorescence, we were able to clearly show that the failure of $\mathrm{HC}$ layer formation is coincident with the loss of Lim1 expression (Fig. 6G,H). In the panel showing the mutant retina, the single Lim $1+$ cell has reached a location that would correspond to the $\mathrm{HC}$ monolayer 
Table 1. Summary of marker analyses

\begin{tabular}{|c|c|c|c|c|c|c|c|c|c|c|c|c|c|c|c|c|c|c|c|c|c|}
\hline & Lim1-LacZ & Calbindin & Prox1 & Pax6 & $D c x$ & Syntaxin-1 & Calretinin & Pep19 & $|s|-1$ & p57 & ChAT & Dab1 & GABA & GLYT-1 & PKCa & Chx10 & Rhodopsin & Recoverin & Sox9 & p27 & Brn3b \\
\hline Horizontal & + & + & + & + & + & + & - & - & - & - & - & - & - & - & - & - & - & - & - & - & - \\
\hline Amacrine & - & + & + & + & + & + & + & + & + & + & + & + & + & + & - & - & - & - & - & - & - \\
\hline Bipolar & - & - & + & - & - & - & - & + & + & - & - & - & - & - & + & + & - & + & - & - & - \\
\hline Ganglion & - & + & - & + & + & - & + & + & + & - & - & - & - & - & - & - & - & - & - & - & + \\
\hline Müller glia & - & - & - & - & - & - & - & - & - & - & - & - & - & - & - & - & - & - & + & + & - \\
\hline Photoreceptors & - & - & - & - & - & - & - & - & - & - & - & - & - & - & - & - & + & + & - & - & - \\
\hline Ectopic LacZ + cells & + & + & + & + & + & + & - & - & - & - & - & - & - & - & - & - & - & - & - & - & - \\
\hline
\end{tabular}

Summarized above are the results of all colocalization studies performed on Lim1 conditional mutant retinas and controls. Colocalization or lack of colocalization between anti-lacZ and a battery of retinal markers are indicated by pluses or minuses, respectively. Cumulatively, these results suggest that the ectopic Lim1-lacZ + cells are horizontal cells in their identity. See the supplement file for an antibody list containing the appropriate references documenting retinal cell type specificity.

(Fig. 6H, arrowhead), whereas all other cells that failed to move in toward the onbl do not express Lim1 (Fig. $6 \mathrm{H}$, arrows). At P3 (Fig. 6I,J), aberrant dendritic growth into the IPL can first be detected (Fig. 6J, arrow), and by P7 these processes are further elaborated (Fig. $6 K, L$ ). Also, in the $\mathrm{P} 3$ example shown in Figure 6J, three lac $\mathrm{Z}+$ cells appear localized near or within the GCL. Although we occasionally did observe ectopic cells within the GCL, the vast majority of Lim1-null lacZ+ cells were located within the lower INL. In conclusion, this time course for the appearance of ectopic Lim1-null, lacZ + HCs, coincident with the time course for Lim1 expression during normal development, is consistent with the hypothesis that Lim1 regulates the ultimate laminar positioning of HCs.

\section{Discussion}

\section{Lamination of the retina}

Because of the temporal overlap in the genesis of retinal cell types (Young, 1985a,b; Cepko et al., 1996; Livesey and Cepko, 2001), migration of a particular retinal cell type occurs concurrently with specification and subsequent movement of other retinal cell types. Thus, the process of retinal neuron migration must be a highly coordinated and directed event enabling the migrating neuron to traverse a dynamic environment on its way to the appropriate retinal layer where it will ultimately establish precise connections within the retinal circuitry. Understanding of this very important event of retinal development may also shed light on basic, conserved events in CNS neuronal migration at large. Despite the importance of understanding the mechanisms of retinal neuron migration and resulting lamination, identification of
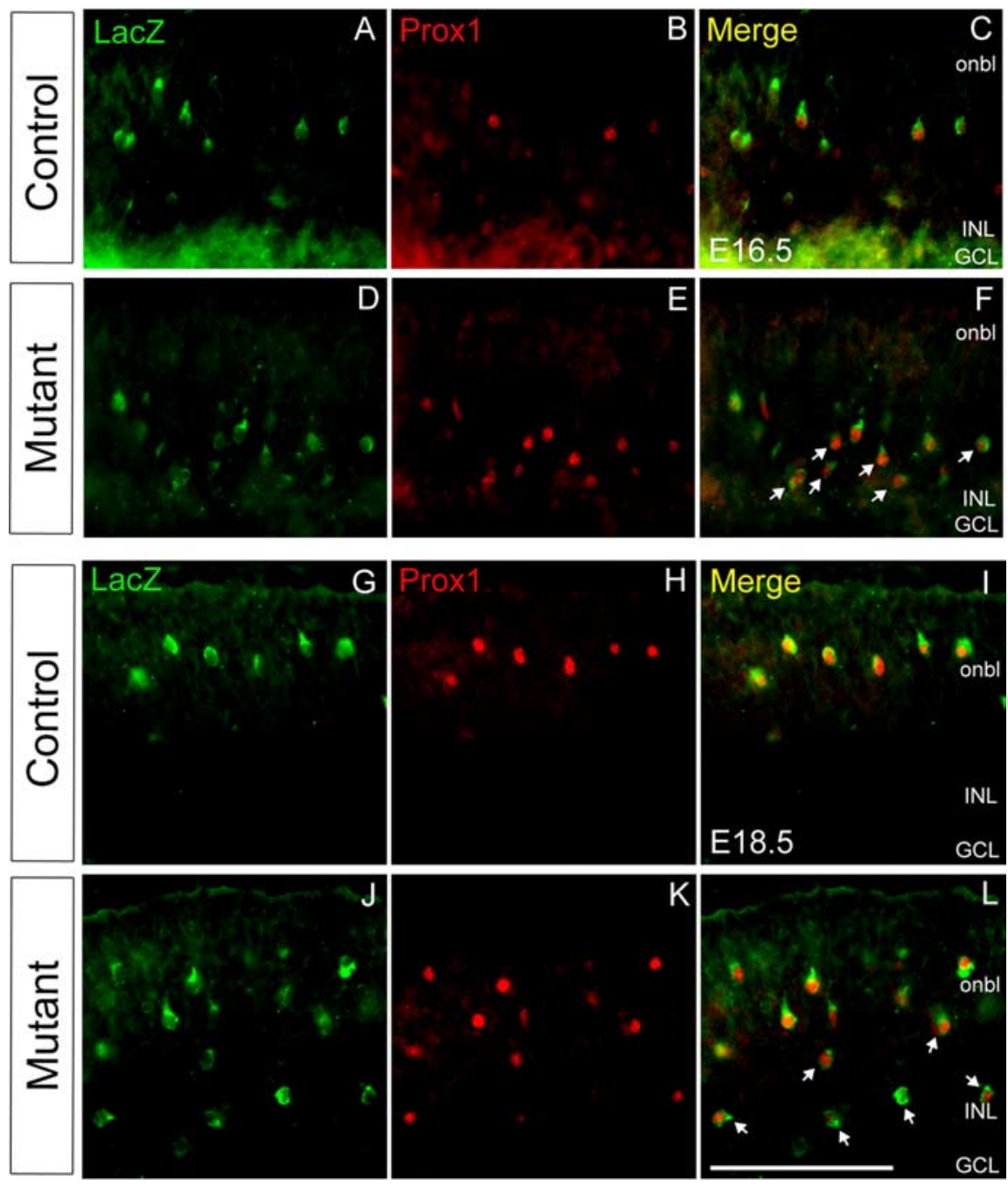

Figure 5. $\boldsymbol{A}-\boldsymbol{F}$, Embryonic Lim1 mutant cells maintain expression of horizontal cell fate determining factor Prox1. At E16.5, control and ectopic lacZ+ cells label with anti-Prox1 antibodies (arrows). G-L, By E18.5, in the control retina, the lacZ/Prox1+ horizontal cells have reached the onbl, whereas this has not properly occurred in the Lim 1-mutant retina. However, the ectopic cells maintain Prox1 expression ( $\boldsymbol{L}$, arrows). The persistence of Prox1 expression in the mutant lac $Z+$ cells suggests appropriate specification to the horizontal cell fate. Scale bar, $50 \mu \mathrm{m}$.

molecular regulators of these processes is lacking. Here, we report $\operatorname{Lim} 1$ as the first identified transcription factor necessary for appropriate laminar positioning of a specific retinal neuron population.

\section{Lim1-lacZ is expressed in migratory horizontal cells}

Horizontal cell development, from fate determination to laminar positioning, is a substantially protracted process. HCs become postmitotic between E11 and E16 (Young, 1985a), but it is not until P3 that all HCs become localized to the HC monolayer. Several groups have interpreted this latency to target the appropriate lamina as a substantial delay in migration toward the prospective HC layer (Hinds and Hinds, 1979; Liu et al., 2000). Recently, by studying the chicken embryo, postmitotic $\mathrm{HC}$ precursors were shown to undergo a bidirectional mode of migration. Instead of migrating directly toward the prospective $\mathrm{HC}$ 

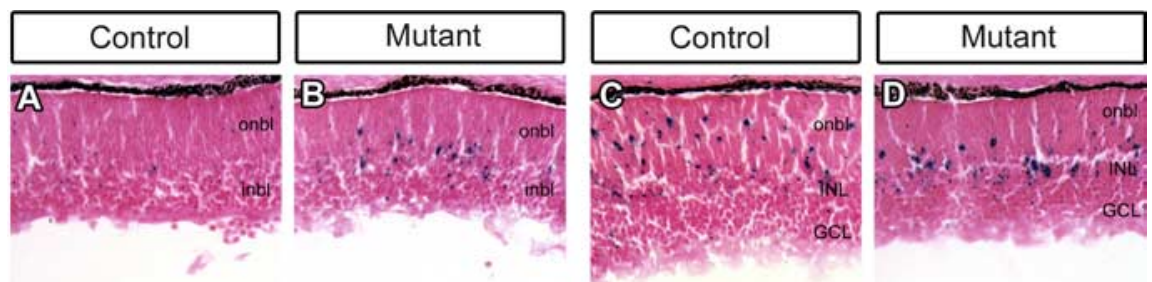

E15.5

E15.5

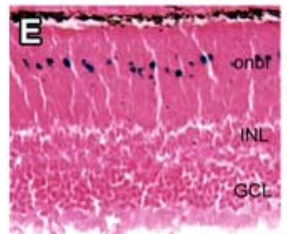

E18.5
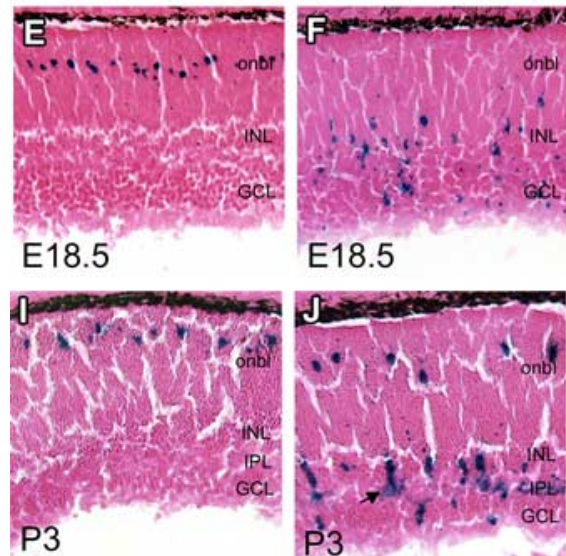

E18.5

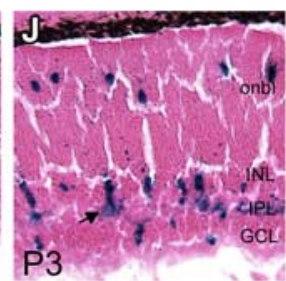

E16.5
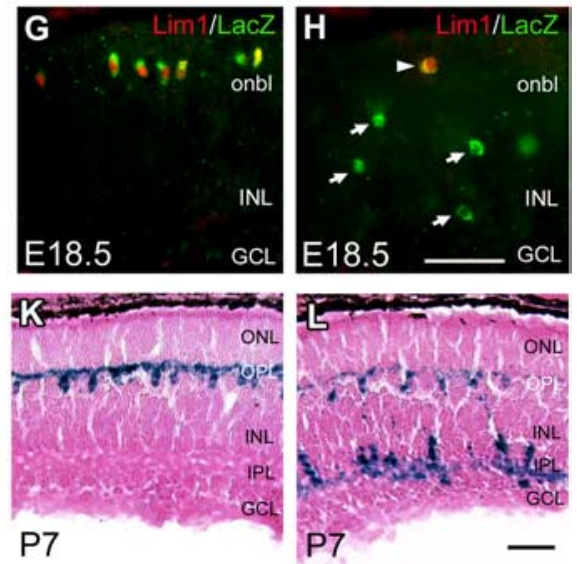

Figure 6. $\boldsymbol{A}, \boldsymbol{B}$, Time course of the $\operatorname{Lim} 1$ conditional mutant horizontal cell migration defect. X-gal staining reveals that the Lim1-lacZ + cell ectopia is first apparent by E15.5. C, D, A day later, the ectopic lacZ+ cells are more numerous with most accumulating within the developing INL and not locating to the onbl as in the control. $\boldsymbol{E}, \boldsymbol{F}$, By E18.5, there is a substantial failure of lacZ + cells to locate to the onbl. $\mathbf{G}, \boldsymbol{H}$, Colabeling for Lim1 and Lim1-lacZ reveals that cells that fail to localize to the onbl do not express Lim1 ( $\boldsymbol{H}$, arrows), whereas a single cell that has migrated to the onbl expresses Lim1, as do cells in the control retina ( $\boldsymbol{G}$, $\boldsymbol{H}$, arrowhead). $\boldsymbol{I}, \boldsymbol{J}, A t P 3$, the ectopic cells project into the developing IPL (J, arrow). $\boldsymbol{K}, \boldsymbol{L}$, By P7, the ectopic cell stratifications are further elaborated. Scale bars: (in $\boldsymbol{L}) \boldsymbol{A}-\boldsymbol{F}, \boldsymbol{I}-\boldsymbol{L}, 50 \mu \mathrm{m}$; (in $\boldsymbol{H}) \boldsymbol{G}, \boldsymbol{H}, 50 \mu \mathrm{m}$.

layer, newly postmitotic HC precursors bypass the prospective $\mathrm{HC}$ layer and instead migrate vitreally toward the developing GCL. After this first phase of migration, the HCs then migrate back to the onbl, thereby arriving at their final resting place (Edqvist and Hallbook, 2004). The apparent lingering developmental process reported by other groups may simply reflect the amount of time it takes the HC progenitor to migrate bidirectionally. In the present study, the Lim1-lacZ allele was not detected until E14.5 and did not become widespread until E15.5. To reiterate, this time point is over $3 \mathrm{~d}$ after HCs first begin to exit the cell cycle. Furthermore, at E15.5, Lim1 + HCs do not express cell cycle marker Ki67 and are thus HC precursors that have already exited the cell cycle. Between E15.5 and E16.5, the Lim1-lacZ+ HCs were scattered throughout the developing retina with a small proportion of these cells localizing to the onbl. We interpret these data as reflecting Lim-lacZ + cell migration to the prospective $\mathrm{HC}$ layer. The observations in these studies place Lim1 expression, and presumably function, at a time when most HCs are already specified and are migrating to the HC layer.

The Lim1 retina-specific mutant provides functional evidence for a bidirectional model of horizontal cell migration

To further investigate the role of Lim1 in HC development, we engineered Lim 1 retina-specific mutants. From these data, we propose a modification to the model of $\mathrm{HC}$ bidirectional migration to include Lim1 as a key regulator (Edqvist and Hallbook, 2004). On exiting the cell cycle, beginning around E11, HC precursors migrate past the future HC layer toward the developing GCL. Subsequently, the HCs migrate away from the vitreal side of the retina toward the future HC layer within the onbl. Adult

Lim1-null, lacZ + cells retained their HC identity, but remain positioned within the innermost regions of the INL, subsequently stratifying within the IPL. Thus, we propose that these mutant HCs successfully complete the first phase of migration toward the GCL, but are unable to complete the second phase of migration toward the onbl. Furthermore, because of the appropriate expression of the $\mathrm{HC}$ fate determining factor Prox1 in E16.5 and E18.5 Lim1 mutant cells, we provide additional support to the idea that Lim1 does not function in $\mathrm{HC}$ fate determination, but rather is essential for HC layer formation. We conclude that the ultimate positioning of HCs from the GCL to the prospective $\mathrm{HC}$ layer, that is, this second migratory phase of the HC, is a Lim1-dependent event (Fig. 7).

Additional support for the idea that $\mathrm{HC}$ migration is indeed a two-step process is our finding that ectopic Lim1-null HC positioning occurs specifically within the lower inner nuclear layer rather than being scattered randomly throughout the retina. These data suggest a general model for INL lamination whereby both $\mathrm{HC}$ and amacrine cell precursors initially respond to localization cue(s) that direct them vitreally toward the developing INL. Subsequently, HCs must overcome this initial signaling mechanism, via Lim1 function, thereby resulting in terminal lamination. Recent research on retinal interkinetic nuclear migration fits within our model of Lim 1 function. Here, through time lapse imaging in zebrafish, it was shown that retinal progenitor cells exhibit a great deal of heterogeneity in nuclear migration parameters that correlates with neurogenic versus proliferative cell divisions. This diversity in cell behavior was suggested as a mechanism whereby otherwise equivalent groups of cells acquire diversity in cell fate and differentiation (Baye and Link, 2007 a,b). Although these studies specifically addressed mitotic retinal progenitor cells, the general conclusions might also be applied to postmitotic, migratory precursor cells. Furthermore, it is important to consider that additional transcription factors or signaling cues might be needed to stop the horizontal cell once it reaches the correct lamina. The components of such a mechanism are currently unknown.

Originally, $\operatorname{Lim} 1$ was identified as a transcriptional regulator of the embryonic head organizer because mice null for Lim1 exhibit a total absence of anterior head structures, including the eyes (Shawlot and Behringer, 1995). Lim1 was also discovered to be vital in determining the choice of spinal cord lateral motor column axon trajectory into the dorsal developing limb (Kania et al., 2000). Very recently, Lim1 and related LIM-homeobox gene Lhx 5 were found to play a role in maintaining the GABAergic identity of dorsal spinal cord interneurons as well as essential factors for cerebellar Purkinje cell differentiation (Pillai et al., 2007; Zhao et al., 2007). The above-mentioned studies are informative because they illustrate examples of LIM-homeobox genes directing neuronal morphology, behavior, or differentiation without influencing initial neuronal fate specification. We propose a comparable role for Lim 1 as a regulator of HC laminar 
positioning rather than a fate determination factor (supplemental Fig. 10, available at www.jneurosci.org as supplemental material). It will also be useful to determine whether other Lhx family members are expressed in migratory retinal neurons other than HCs. Indeed, recent publications report the expression of $L h x 2, L h \times 3, L h x 4$, and $\operatorname{Lh} x 9$ within the adult retina (Zhang et al., 2005, 2006). Detailed temporal and spatial expression profiles for these family members remain to be determined. However, a LIM-code directing cell typespecific retinal layer formation may very well exist.

\section{Morphological plasticity of horizontal cell precursors}

In the absence of Lim1 function, HCs remain within the inner aspects of the INL among the amacrine cells. In this ectopic location, the mutant HCs exhibit a differentiated morphology reminiscent of amacrine cells. However, the ectopic cells express none of the amacrine cell-specific markers tested. By P3, these lacZ+ cells project into the IPL and eventually stratify in the innermost portion of the IPL adjacent to the GCL. Our interpretation of these data is that misplaced HC precursors are influenced by differentiation cues residing within the ectopic environment, thereby causing these cells to adopt a morphology consistent with neighboring cell types (i.e., amacrine cells). This conclusion would suggest that postmitotic horizontal neurons remain quite plastic and that ultimate cellular morphology is not a fixed state. Consistent with this idea is evidence showing that genetic respecification of all rods to cones, or all cones to rods, modulates HC dendritic (and axonal) morphologies accordingly (Raven et al., 2007). Also, using mosaic deletion of Lim 1 in the retina, we analyzed the effects of decreased HC density on the remaining, normally localized, Lim $1+$ HCs. These data reveal that HC dendritic field size, within a depleted HC layer, is expanded by nearly $50 \%$, thereby providing additional support that HC morphology is influenced by local environmental cues (R. A. Poché, unpublished observations). These studies highlight the ability of normally placed, postmitotic HCs to respond to local environmental cues that modulate normal HC morphology. This present study suggests that $\mathrm{HC}$ plasticity is even more robust than originally suggested because ectopically localized HCs can adopt an entirely new morphology. In light of very recent successes in transplanting newly postmitotic photoreceptor precursors into adult retinas (MacLaren et al., 2006), our findings of HC plasticity may be of particular interest for understanding the cellular properties that confer the capacity for retinal repair.

Our study of the role of Lim1 in HC positioning has established an entry point to delineate genetic pathways controlling retinal lamination. Based on the identity of Lim 1 as a transcriptional regulator, Lim1 likely controls gene products that may be vital for cell motility. Furthermore, because Lim1 is also expressed in various locations throughout the developing CNS, it may also play a more global role in neuronal layer formation (K. M. Kwan and R. R. Behringer, unpublished observations).

\section{References}

Baye LM, Link BA (2007a) Interkinetic nuclear migration and the selection of neurogenic cell divisions during vertebrate retinogenesis. J Neurosci 27:10143-10152.

Baye LM, Link BA (2007b) Nuclear migration during retinal development. Brain Res, in press.

Cepko CL (1999) The roles of intrinsic and extrinsic cues and bHLH genes in the determination of retinal cell fates. Curr Opin Neurobiol 9:37-46.

Cepko CL, Austin CP, Yang X, Alexiades M, Ezzeddine D (1996) Cell fate determination in the vertebrate retina. Proc Natl Acad Sci USA 93:589-595.

Dyer MA, Livesey FJ, Cepko CL, Oliver G (2003) Prox1 function controls progenitor cell proliferation and horizontal cell genesis in the mammalian retina. Nat Genet 34:53-58.

Edqvist PH, Hallbook F (2004) Newborn horizontal cells migrate bidirectionally across the neuroepithelium during retinal development. Development 131:1343-1351.

Fu X, Sun H, Klein WH, Mu X (2006) Beta-catenin is essential for lamination but not neurogenesis in mouse retinal development. Dev Biol 299:424-437.

Fujitani Y, Fujitani S, Luo H, Qiu F, Burlison J, Long Q, Kawaguchi Y, Edlund H, Macdonald RJ, Furukawa T, Fujikado T, Magnuson MA, Xiang M, Wright CV (2006) Ptfla determines horizontal and amacrine cell fates during mouse retinal development. Development 133:4439-4450.

Furuta Y, Lagutin O, Hogan BL, Oliver GC (2000) Retina- and ventral forebrain-specific Cre recombinase activity in transgenic mice. Genesis 26:130-132.

Haeseleer F, Imanishi Y, Maeda T, Possin DE, Maeda A, Lee A, Rieke F, Palczewski K (2004) Essential role of $\mathrm{Ca}^{2+}$-binding protein 4, a Cav1.4 
channel regulator, in photoreceptor synaptic function. Nat Neurosci 7:1079-1087.

Hatakeyama J, Kageyama R (2004) Retinal cell fate determination and bHLH factors. Semin Cell Dev Biol 15:83-89.

Haverkamp S, Wassle H (2000) Immunocytochemical analysis of the mouse retina. J Comp Neurol 424:1-23.

Hinds JW, Hinds PL (1979) Differentiation of photoreceptors and horizontal cells in the embryonic mouse retina: an electron microscopic, serial section analysis. J Comp Neurol 187:495-511.

Johnson DA, Donovan SL, Dyer MA (2006) Mosaic deletion of Rb arrests rod differentiation and stimulates ectopic synaptogenesis in the mouse retina. J Comp Neurol 498:112-128.

Kania A, Johnson RL, Jessell TM (2000) Coordinate roles for LIM homeobox genes in directing the dorsoventral trajectory of motor axons in the vertebrate limb. Cell 102:161-173.

Kobayashi A, Shawlot W, Kania A, Behringer RR (2004) Requirement of Lim1 for female reproductive tract development. Development 131:539-549.

Kobayashi A, Kwan KM, Carroll TJ, McMahon AP, Mendelsohn CL, Behringer RR (2005) Distinct and sequential tissue-specific activities of the LIM-class homeobox gene Lim1 for tubular morphogenesis during kidney development. Development 132:2809-2823.

Koike C, Nishida A, Akimoto K, Nakaya MA, Noda T, Ohno S, Furukawa T (2005) Function of atypical protein kinase C lambda in differentiating photoreceptors is required for proper lamination of mouse retina. J Neurosci 25:10290-10298.

Kwan KM, Behringer RR (2002) Conditional inactivation of Liml function. Genesis 32:118-120.

Lee EJ, Kim IB, Lee E, Kwon SO, Oh SJ, Chun MH (2003) Differential expression and cellular localization of doublecortin in the developing rat retina. Eur J Neurosci 17:1542-1548.

Li S, Mo Z, Yang X, Price SM, Shen MM, Xiang M (2004) Foxn4 controls the genesis of amacrine and horizontal cells by retinal progenitors. Neuron 43:795-807.

Liu W, Wang JH, Xiang M (2000) Specific expression of the LIM/homeodomain protein Lim-1 in horizontal cells during retinogenesis. Dev Dyn 217:320-325.

Livesey FJ, Cepko CL (2001) Vertebrate neural cell-fate determination: lessons from the retina. Nat Rev Neurosci 2:109-118.

MacLaren RE, Pearson RA, MacNeil A, Douglas RH, Salt TE, Akimoto M, Swaroop A, Sowden JC, Ali RR (2006) Retinal repair by transplantation of photoreceptor precursors. Nature 444:203-207.

Malicki J, Neuhauss SC, Schier AF, Solnica-Krezel L, Stemple DL, Stainier DY, Abdelilah S, Zwartkruis F, Rangini Z, Driever W (1996) Mutations affecting development of the zebrafish retina. Development 123:263-273.
Malicki J, Jo H, Pujic Z (2003) Zebrafish N-cadherin, encoded by the glass onion locus, plays an essential role in retinal patterning. Dev Biol 259:95-108.

Masai I, Lele Z, Yamaguchi M, Komori A, Nakata A, Nishiwaki Y, Wada H, Tanaka H, Nojima Y, Hammerschmidt M, Wilson SW, Okamoto H (2003) N-cadherin mediates retinal lamination, maintenance of forebrain compartments and patterning of retinal neurites. Development 130:2479-2494.

Ohtoshi A, Justice MJ, Behringer RR (2001) Isolation and characterization of Vsx1, a novel mouse CVC paired-like homeobox gene expressed during embryogenesis and in the retina. Biochem Biophys Res Commun 286:133-140.

Pillai A, Mansouri A, Behringer R, Westphal H, Goulding M (2007) Lhx1 and Lhx5 maintain the inhibitory-neurotransmitter status of interneurons in the dorsal spinal cord. Development 134:357-366.

Raven MA, Oh EC, Swaroop A, Reese BE (2007) Afferent control of horizontal cell morphology revealed by genetic respecification of rods and cones. J Neurosci 27:3540-3547.

Rice DS, Curran T (2000) Disabled-1 is expressed in type AII amacrine cells in the mouse retina. J Comp Neurol 424:327-338.

Rowan S, Cepko CL (2004) Genetic analysis of the homeodomain transcription factor Chx10 in the retina using a novel multifunctional BAC transgenic mouse reporter. Dev Biol 271:388-402.

Seymour PA, Freude KK, Tran MN, Mayes EE, Jensen J, Kist R, Scherer G, Sander M (2007) SOX9 is required for maintenance of the pancreatic progenitor cell pool. Proc Natl Acad Sci USA 104:1865-1870.

Shawlot W, Behringer RR (1995) Requirement for Lim1 in head-organizer function. Nature 374:425-430.

Wang SW, Kim BS, Ding K, Wang H, Sun D, Johnson RL, Klein WH, Gan L (2001) Requirement for math5 in the development of retinal ganglion cells. Genes Dev 15:24-29.

Young RW (1985a) Cell differentiation in the retina of the mouse. Anat Rec 212:199-205.

Young RW (1985b) Cell proliferation during postnatal development of the retina in the mouse. Brain Res 353:229-239.

Zhang SS, Xu X, Li J, Liu MG, Zhao H, Soares MB, Barnstable CJ, Fu XY (2005) Comprehensive in silico functional specification of mouse retina transcripts. BMC Genomics 6:40.

Zhang SS, Xu X, Liu MG, Zhao H, Soares MB, Barnstable CJ, Fu XY (2006) A biphasic pattern of gene expression during mouse retina development. BMC Dev Biol 6:48.

Zhao Y, Kwan KM, Mailloux CM, Lee WK, Grinberg A, Wurst W, Behringer RR, Westphal H (2007) LIM-homeodomain proteins Lhx1 and Lhx5, and their cofactor Ldb1, control Purkinje cell differentiation in the developing cerebellum. Proc Natl Acad Sci USA 104:13182-13186. 